\title{
The CHASE laboratory search for chameleon dark energy
}

\author{
Jason H. STEFFEN* \\ Fermilab Center for Particle Astrophysics \\ E-mail: jsteffenefnal.gov
}

\section{For the GammeV-CHASE collaboration}

\begin{abstract}
A scalar field is a favorite candidate for the particle responsible for dark energy. However, few theoretical means exist that can simultaneously explain the observed acceleration of the Universe and evade tests of gravity. The chameleon mechanism, whereby the properties of a particle depend upon the local environment, is one possible avenue. We present the results of the Chameleon Afterglow Search (CHASE) experiment, a laboratory probe for chameleon dark energy. CHASE marks a significant improvement over other searches for chameleons both in terms of its sensitivity to the photon/chameleon coupling as well as its sensitivity to the classes of chameleon dark energy models and standard power-law models. Since chameleon dark energy is virtually indistinguishable from a cosmological constant, CHASE tests dark energy models in a manner not accessible to astronomical surveys.
\end{abstract}

35th International Conference of High Energy Physics - ICHEP2010,

July 22-28, 2010

Paris France

${ }^{*}$ Speaker. 


\section{Introduction}

A variety of observational evidence indicates that the expansion of the universe is accelerating, for which a promising class of explanations is scalar field "dark energy" with negative pressure [1]. There are only three known ways to hide such scalar fields from existing experimental tests: weak or pseudoscalar couplings between dark energy and matter [2]; effectively weak couplings locally [3]; and an effectively large field mass locally, as in chameleon theories [4, 5, 6].

Chameleons are scalar (or pseudoscalar) fields with a nonlinear potential and a coupling to the local energy density. They increase their effective mass in high-density environments, while remaining light in the intergalactic medium. Photon-coupled chameleons may be detected through laser experiments [9] or in radio frequency cavities [10]. In laser experiments, photons travelling through a vacuum chamber immersed in a magnetic field oscillate into chameleons. They are then trapped through the chameleon mechanism by the dense walls and windows of the chamber $[9,11,12]$. After a population of chameleons is produced, the laser is turned off and a photodetector exposed in order to observe the photon afterglow as trapped chameleons oscillate back to photons. The GammeV Chameleon Afterglow Search (CHASE) is an experiment to search for photon coupled chameleons [13]. Its results improve upon the original GammeV experiment [9] with better sensitivity to both matter and photon couplings to chameleons, and sensitivity to a broader class of chameleon models.

We consider actions of the form

$$
S=\int d^{4} x \sqrt{-g}\left(\frac{1}{2} M_{\mathrm{Pl}}^{2} R-\frac{1}{2} \partial_{\mu} \phi \partial^{\mu} \phi-V(\phi)-\frac{1}{4} e^{\beta_{\gamma} \phi / M_{\mathrm{Pl}}} F^{\mu v} F_{\mu v}+\mathscr{L}_{\mathrm{m}}\left(e^{2 \beta_{\mathrm{m}} \phi / M_{\mathrm{Pl}}} g_{\mu v}, \psi_{\mathrm{m}}^{i}\right)\right)
$$

where the reduced Planck mass $M_{\mathrm{Pl}}=2.43 \times 10^{18} \mathrm{GeV}, \mathscr{L}_{\mathrm{m}}$ the Lagrangian for matter fields $\psi_{\mathrm{m}}^{i}$, and $\beta_{\gamma}$ and $\beta_{\mathrm{m}}$ are dimensionless chameleon couplings to photons and matter respectively. The dynamics of this field are governed by an effective potential that depends on a potential $V(\phi)$, the background matter density $\rho_{\mathrm{m}}$, and the electromagnetic field Lagrangian density $\rho_{\gamma}=F^{\mu v} F_{\mu \nu} / 4=$ $\left(B^{2}-E^{2}\right) / 2$ (for pseudoscalars $\rho_{\gamma}=F^{\mu v} \tilde{F}_{\mu v} / 4=\vec{B} \cdot \vec{E}$ ):

$$
V_{\mathrm{eff}}(\phi, \vec{x})=V(\phi)+e^{\frac{\beta_{\mathrm{m}} \phi}{M_{\mathrm{Pl}}}} \rho_{\mathrm{m}}(\vec{x})+e^{\frac{\beta_{\gamma \phi} \phi}{M_{\mathrm{Pl}}}} \rho_{\gamma}(\vec{x})
$$

A well-studied class of chameleon models has a potential of the form [6]

$$
V(\phi)=M_{\Lambda}^{4} e^{\kappa\left(\frac{\phi}{M_{\Lambda}}\right)^{N}} \approx M_{\Lambda}^{4}\left[1+\kappa\left(\frac{\phi}{M_{\Lambda}}\right)^{N}\right] .
$$

where $N$ is a real number and $M_{\Lambda}=\rho_{\mathrm{de}}^{1 / 4} \approx 2.4 \times 10^{-3} \mathrm{eV}$ is the mass scale of the dark energy density $\rho_{\mathrm{de}}$ and $\kappa$ is a dimensionless constant. The constant term in this potential causes cosmic acceleration that is indistinguishable from a cosmological constant for cosmological surveys. However, the local dynamics from the power-law term can be probed in the laboratory.

The conversion probability between photons and chameleons is

$$
\mathscr{P}_{\gamma \leftrightarrow \phi}=\left(\frac{2 \omega \beta_{\gamma} B}{M_{\mathrm{P} 1} m_{\mathrm{eff}}^{2}}\right)^{2} \sin ^{2}\left(\frac{m_{\mathrm{eff}}^{2} \ell}{4 \omega}\right) \hat{k} \times(\hat{x} \times \hat{k}) .
$$


Here, $\omega$ is the particle energy, $m_{\mathrm{eff}}=\sqrt{V_{\mathrm{eff}, \phi \phi}}$ is the effective chameleon mass in the environment, $\ell$ is the distance travelled through the magnetic field, and $\hat{k}$ is the particle direction. A single parameter $\eta$ can be used to describe the chameleon effect. If the chameleon mass in the chamber is dominated by the matter coupling, then $m_{\mathrm{eff}} \propto \rho_{\mathrm{m}}^{\eta}$ where $\eta=(N-2) /(2 N-2)$ [14].

When a photon/chameleon wavefunction strikes an opaque surface, there is a model-dependent phase shift $\xi_{\text {ref }}$ between the two components and a small absorption of photon amplitude due. On the other hand, a glass window performs a quantum measurement—chameleons reflect while photons are transmitted. The decay rate of a chameleon to a photon $\Gamma_{\mathrm{dec}, \gamma}$, is found by averaging over initial directions and positions. The observable afterglow rate per chameleon $\Gamma_{\text {aft }}$ is found by averaging over those trajectories that allow a photon to reach the detector.

\section{Experimental Approach}

The design of the CHASE apparatus is shown in Fig. 1. In addition to the windows at the ends of the vacuum chamber, we centered two glass windows in the magnet which divide the magnetic field into three partitions of lengths $1.0 \mathrm{~m}, 0.3 \mathrm{~m}$, and $4.7 \mathrm{~m}$. The shorter partition lengths provide sensitivity to larger-mass chameleons. For a fixed magnetic field there are limits to the smallest and largest detectable $\beta_{\gamma}$-small $\beta_{\gamma}$ produce small afterglow signals while with large $\beta_{\gamma}$ the chameleon population will decay before the detector can be exposed. We improve our sensitivity to large $\beta_{\gamma}$ by operating at a variety of lower magnetic fields, which lengthen the decay time of the chameleon population. The CHASE vacuum system uses ion pumps and cryogenic pumping on the cold $(\sim 4 \mathrm{~K})$ bore of the magnet. This design allows CHASE to probe $\eta$ as low as 0.1 .

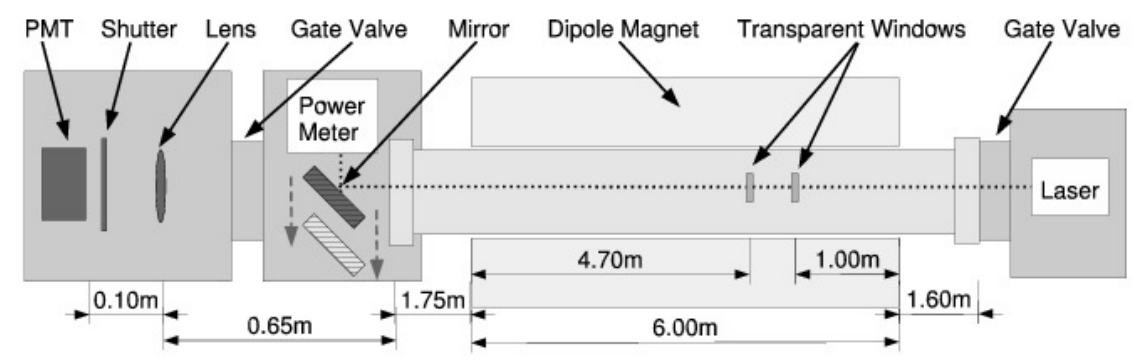

Figure 1: Schematic of the CHASE apparatus.

We collected data in 14 configurations—-seven magnetic field values $(0.050,0.090,0.20,0.45$, 1.0, 2.2, and 5.0 Telsa) and both polarizations of the laser. We repeated measurements at 5.0 T for a total of 16 science "runs". A single science run consists of shining a 3.5 Watt, 532nm laser through the cavity for ten minutes, then exposing the PMT to the apparatus for 14 minutes while cycling a mechanical shutter open and closed in $\sim 15$ second intervals each. At the highest magnetic field the filling and observation stages are extended to 5 hours and 45 minutes respectively. Before and after each science run, there is a 15 minute calibration run which we use to measure the properties of any excess photon rate coming from the apparatus (due in part to discharge from the ion pumps). We see an excess rate of $1.15 \pm 0.08 \mathrm{~Hz}$. Our overal photon detection efficiency is $\varepsilon_{\mathrm{det}}=0.29$.

Following the filling stage, we observe a decaying rate of photons that we call "orange glow". Its cause is unknown, but it has several properties that distinguish it from a chameleon signal: it 
does not appear near the expected 532nm green wavelength of the laser, it is independent of the magnetic field strength and the laser polarization, and the amplitude of the glow depends upon the temperature of the magnet bore- being near zero at room temperature and several hundred $\mathrm{Hz}$ near $4 \mathrm{~K}$. To mitigate its effects, we ignore the initial $\sim 120$ seconds of data from each science run.

\section{Analysis and Results}

For the CHASE geometry, the rates $\Gamma_{\mathrm{dec}, \gamma}$ and $\Gamma_{\text {aft }}$ are computed in [14]. We account for the absorption of photon amplitude and the differences in the induced phase shift $\xi_{\text {ref }}$ between the $s$ and $p$ polarizations at each reflection. Given these rates, the population $N_{\phi}$ of chameleons in the vacuum chamber is found by integrating

$$
\frac{d N_{\phi}}{d t}=F_{\gamma}(t) \mathscr{P}_{\gamma \leftrightarrow \phi}-N_{\phi}(t) \Gamma_{\mathrm{dec}, \gamma}
$$

where $F_{\gamma}(t)$ is the rate that laser photons stream through the chamber. Afterglow photons emerge and hit the PMT at a rate $F_{\text {aft }}(t)=\varepsilon_{\operatorname{det}} N_{\phi}(t) \Gamma_{\text {aft }}$.

Data for all science runs with a given laser polarization and for a set of runs with $\vec{B}=0$ are simultaneously analyzed using the Profile Likelihood method [15]. As nuisance parameters we include a common, exponentially decaying signal, which eliminates the long-decay tail of the orange glow, and each run is allowed an independent constant offset constrained by the possible $0.40 \mathrm{~Hz}$ run-to-run variations in the ion pump glow. We compare the $\chi^{2}$ for the chameleon model with that for the model where no chameleon is present. Any chameleon model whose $\chi^{2}$ is greater by 6.0 is excluded to $95 \%$ confidence.

Analysis of our data shows no evidence for a photon-coupled chameleon. The mean and RMS of the residuals for the no chameleon model are 0.05 and $1.35 \mathrm{~Hz}$ for pseudoscalar couplings $\left(\chi^{2}=421\right.$ with 471 degrees of freedom (DOF)) and 0.06 and $1.62 \mathrm{~Hz}$ for scalar couplings $\left(\chi^{2}=502\right.$ with 472 DOF). Fig. 2 shows parameters excluded to $95 \%$ confidence for scalars and pseudoscalars assuming $m_{\text {eff }}$ dependence on $B$ to be negligible and $\xi_{\text {ref }}=0$. These constraints reach four significant milestones: they bridge the gap between bounds on $\beta_{\gamma}$ from GammeV and from colliders [8], they exclude a range of $\beta_{\gamma}$ spanning four orders of magnitude at masses around the dark energy scale $\left(2.4 \times 10^{-3} \mathrm{eV}\right)$, they rule out photon couplings roughly an order of magnitude below previous limits in this mass range where $\beta_{\gamma}<7.1 \times 10^{10}$ for scalar and $\beta_{\gamma}<7.6 \times 10^{10}$ for pseudoscalar chameleons, and they are sensitive to chameleon dark energy models and chameleon power-law models where $\eta>0.1$, including $V \propto \phi^{4}$.

Figure 2 shows CHASE constraints (at 95\%) for select potentials given by Eq. (1.3). These limits truncate at low $\beta_{\mathrm{m}}$ by the requirement that chameleons reflect from the chamber walls, at high $\beta_{\mathrm{m}}$ by destructive interference at large $m_{\text {eff }}$ (see Fig. 2), and at low $\beta_{\gamma}$ by undetectably small signals. Not surprisingly, theories with the largest $\eta$ are excluded over the greatest range of $\beta_{\mathrm{m}}$. These constraints complement those from torsion pendula, which probe $\beta_{\mathrm{m}} \sim 1$, and are consistent with constraints from Casimir force measurements for $N=4$ [7]. CHASE data exclude chameleons spanning five orders of magnitude in photon coupling and over 12 orders of magnitude in matter coupling for individual models. They probe a wide range of chameleon models, and give significantly improved constraints for cosmologially interesting chameleon dark energy models. 

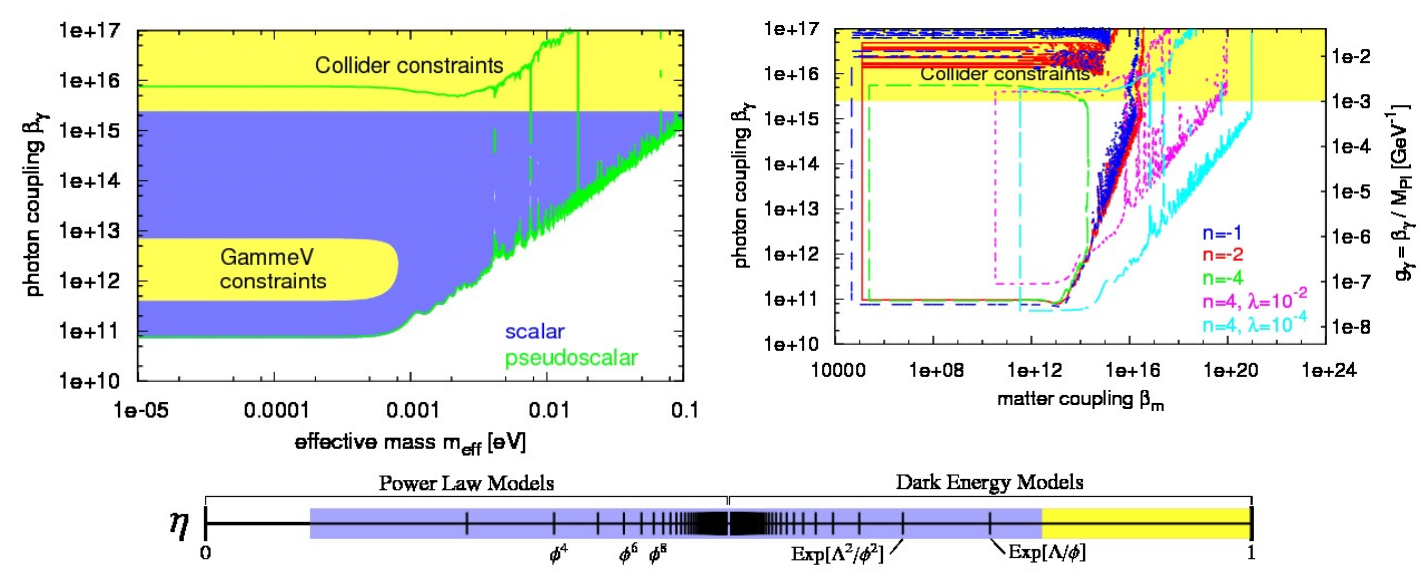

Figure 2: Left: Scalar (solid) and pseudoscalar (outline) constraints, at 95\% confidence, in the $\left(m_{\mathrm{eff}}, \beta_{\gamma}\right)$ plane for $\xi_{\text {ref }}=0$. Right: $95 \%$ confidence-level constraints on chameleons with power law potentials (1.3). For potentials whith $N<0$ we set $\kappa=1$; for $\phi^{4}$ theory $(N=4)$, we use the standard $\kappa=\lambda / 4$ !. Bottom: Chameleon models probed by CHASE as parameterized by $\eta$. GammeV sensitivity is yellow while CHASE sensitivity is blue.

Acknowledgements: This work is supported by the U.S. Department of Energy (No. DE-AC0207CH11359) and the Kavli Institute for Cosmological Physics (NSF PHY-0114422).

\section{References}

[1] R. R. Caldwell, R. Dave, and P. J. Steinhardt. Phys. Rev. Lett., 80:1582, 1998.

[2] J. A. Frieman, C. T. Hill, A. Stebbins, and I. Waga. Phys. Rev. Lett., 75:2077-2080, 1995.

[3] G. R. Dvali, G. Gabadadze, and M. Porrati. Phys. Lett. B, 485:208-214, 2000.

[4] J. Khoury and A. Weltman. Phys. Rev. Lett., 93, 2004. 171104.

[5] J. Khoury and A. Weltman. Phys. Rev. D, 69, 2004. 044026.

[6] Ph. Brax, C. van de Bruck, A.-C. Davis, J. Khoury, and A. Weltman. Phys. Rev. D, 70, 2004. 123518.

[7] P. Brax, C. van de Bruck, A. C. Davis, D. F. Mota, and D. J. Shaw. Phys. Rev. D, 76:124034, 2007. ePrint arXiv:0709.2075.

[8] P. Brax, C. Burrage, A.-C. Davis, D. Seery, and A. Weltman. JHEP, 0909:128, 2009. e-print arXiv:0904.3002.

[9] A. S. Chou et al. Phys. Rev. Lett, 102, 2009. 030402.

[10] G. Rybka et al. Phys. Rev. Lett., 105(5):051801, 2010.

[11] M. Ahlers et al. Phys. Rev. D., 77:015018, 2008.

[12] H. Gies, D. F. Mota, and D. J. Shaw. Phys. Rev. D, 77:025016, 2008.

[13] J. H. Steffen et al. e-print arXiv:1010.0988, 2010.

[14] A. Upadhye, J. H. Steffen, and A. Weltman. Phys. Rev. D, 81:015013, 2010.

[15] W. A. Rolke, A. M. Lopez, and J. Conrad. NIM A, 551:493, 2005.

[16] P. Brax and C. Burrage. 2010. arXiv:1009.1065. 